OPEN ACCESS

Edited by:

Harish Vashisth,

University of New Hampshire, USA

Reviewed by:

Silvina Ponce Dawson,

Universidad de Buenos Aires,

Argentina

Eric R. May,

University of Connecticut, USA

Rohit Rajgaria,

Princeton University, USA

*Correspondence: Chiara Bianca Maria Platania chiara.platania@unict.it; Gabriele Oliva g.oliva@unicampus.it

Specialty section: This article was submitted to Computational Physiology and Medicine, a section of the journal Frontiers in Bioengineering and Biotechnology

Received: 29 May 2015 Accepted: 12 October 2015 Published: 30 October 2015

Citation:

Di Paola L, Platania CBM, Oliva G, Setola R, Pascucci F and Giuliani A (2015) Characterization of protein-protein interfaces through a protein contact network approach.

Front. Bioeng. Biotechnol. 3:170. doi: 10.3389/fbioe.2015.00170

\section{Characterization of protein-protein interfaces through a protein contact network approach}

\author{
Luisa Di Paola ${ }^{1}$, Chiara Bianca Maria Platania ${ }^{2 *}$, Gabriele Oliva ${ }^{1 *}$, Roberto Setola ${ }^{1}$, \\ Federica Pascucci ${ }^{3}$ and Alessandro Giuliani ${ }^{4}$ \\ ${ }^{1}$ Facoltà Dipartimentale di Ingegneria, Università Campus Bio-Medico di Roma, Rome, Italy, ${ }^{2}$ Department of Biomedical and \\ Biotechnological Sciences, University of Catania, Catania, Italy, ${ }^{3}$ Dipartimento di Informatica e Automazione, Università degli \\ studi Roma Tre, Rome, Italy, ${ }^{4}$ Dipartimento di Ambiente e Connessa Prevenzione Primaria, Istituto Superiore di Sanità, \\ Rome, Italy
}

Anthrax toxin comprises three different proteins, jointly acting to exert toxic activity: a non-toxic protective agent (PA), toxic edema factor (EF), and lethal factor (LF). Binding of PA to anthrax receptors promotes oligomerization of PA, binding of EF and LF, and then endocytosis of the complex. Homomeric forms of PA, complexes of PA bound to LF and to the endogenous receptor capillary morphogenesis gene 2 (CMG2) were analyzed. In this work, we characterized protein-protein interfaces (PPIs) and identified key residues at PPIs of complexes, by means of a protein contact network (PCN) approach. Flexibility and global and local topological properties of each PCN were computed. The vulnerability of each PCN was calculated using different node removal strategies, with reference to specific PCN topological descriptors, such as participation coefficient, contact order, and degree. The participation coefficient $P$, the topological descriptor of the node's ability to intervene in protein inter-module communication, was the key descriptor of PCN vulnerability of all structures. High $P$ residues were localized both at PPIs and other regions of complexes, so that we argued an allosteric mechanism in protein-protein interactions. The identification of residues, with key role in the stability of PPIs, has a huge potential in the development of new drugs, which would be designed to target not only PPIs but also residues localized in allosteric regions of supramolecular complexes.

Keywords: anthrax toxin, protein-protein interactions, protein contact networks, network resilience

\section{INTRODUCTION}

Protein-protein interactions play a key role in the biological signaling pathways; however, they were considered difficult to study in terms of their chemo-physical and structural properties. For a long time, scientists simply relied on the functional properties of protein complexes, disregarding the physical details of protein-protein interactions. This restriction had drastically limited the therapeutic interventions impinging on such interactions. This situation changed because of increased structural information; therefore, drugs specifically designed to target protein-protein interactions have reached the market (Arkin et al., 2014).

Once key residues (hot-spot residues) at protein-protein interfaces (PPIs) are identified, PPIs become challenging pharmacological targets with a huge potential in structure-based drug design (Wells and McClendon, 2007). Hot-spot residues largely contribute to the protein-protein binding 
energy and could be spread over wider "cold regions," which comprise residues slightly contributing to complex stability (Clackson and Wells, 1995). Hot-spot residues are experimentally identified by mutating residues to alanine: hot spots are those residues that, if mutated, lead to a change in protein-protein binding energy $>2.0 \mathrm{kcal} / \mathrm{mol}$ (Thorn and Bogan, 2001).

Experimental methods are generally expensive and timeconsuming, and predictive computational methods may largely reduce time and cost by guiding the mutagenesis of putative hotspot residues (Morrow and Zhang, 2012). Computational methods for prediction of hot-spot residues include computational alanine scanning, molecular dynamics, and machine learningbased methods (Morrow and Zhang, 2012); however, often it is necessary to combine methods in order to reach successful predictions.

The computational approach based on protein contact networks (PCNs) has been recently applied to study protein complexes (Di Paola et al., 2015). Although PCN is a minimalist tool, it is an efficient way to study protein structure-function relationships (Di Paola et al., 2013; Cheng et al., 2015). PCN formalism considers protein structures as networks whose nodes are amino acid residues, and two nodes (residues) are in contact if their distance in the 3D structure is within $4-8 \AA$. PCN helps in elucidating key processes, such as folding (Plaxco et al., 2000) and ligand binding (De Ruvo et al., 2012). Additional methods are based on centrality metrics and graph-spectral clustering, which are based on network partition into clusters of highly interconnected residues, through the analysis of global properties of PCNs (Brinda and Vishveshwara, 2005). PCN is also effective in the localization of hot-spot residues, by means of identification of highly connected hubs (residues) and clusters of nodes (residues), which corresponds to evolutionary conserved residues (Karain and Qaraeen, 2015). Furthermore, analysis of molecular dynamics, based on elastic network models, revealed that hotspot residues show a moderate-high flexibility, which accounts for conformational accommodation upon binding (Chennubhotla et al., 2005).

Indeed, PCN approach has a high potential of applicability to the study of protein structures by means of simplified representations, e.g., networks and cluster maps. Therefore, we hereby propose an approach that links topology of PPIs to complex stability through the PCN formalism. We applied this method to complexes of protective agent (PA) bounded with lethal factor (LF) and CMG2. Furthermore, we analyzed monomeric and octameric PA. The analysis of PPIs for these complexes will open up the possibility to develop anthrax antidotes and possibly new anti-angiogenic agents.

The attention on anthrax toxicity raised when, in 2001, 22 people were exposed to mail envelopes contaminated by anthrax spores (Jernigan et al., 2001, 2002; Goel, 2015). The possibility of anthrax diffusion induced a widespread terror in the population, calling for huge attention from public authorities (Wein et al., 2003). Anthrax could be easily spread as bio-weapon, because of its low cost and high stability; moreover, anthrax spores can cause different forms of infection with a high mortality rate.

The molecular machinery behind the Bacillus anthracis toxicity relies on a trimeric protein complex (Young and Collier, 2007), which is composed of PA, LF, and edema factor (EF). Anthrax exerts its toxicity through the following steps: PA binding to extracellular domain of anthrax receptors (ANTXRs), PA oligomerization, binding of EF and LF, and endocytosis. EF and LF translocation through the PA pre-pore is promoted by low $\mathrm{pH}$ in the endosome (Young and Collier, 2007). So far, two ANTXRs have been cloned: the tumor endothelial marker 8 or anthrax receptor 1 (TEM8/ANTXR1) and the CMG2, also named as ANTXR2.

Toxicity of PA is mainly related to the activation of CMG2 receptor, because of its wider expression and higher affinity for PA compared to the TEM8 receptor. A key residue, leucine 56 in TEM8 mutated into alanine in CMG2, seems to influence PA affinity for ANTXR1 receptor (Fu et al., 2010). Indeed, the designing of drugs, which target PA/LF and PA/ANTXRs interfaces, could represent a key step in the development of an anthrax antidote. Furthermore, TEM8 and CMG2 receptors play a role in epithelial and endothelial cell functions, so that mutations of TEM8 and CMG2 lead to very rare diseases, whose pathological mechanism is still largely unknown (Deuquet et al., 2011). TEM8 is involved in the regulation of expression of vascular endothelial growth factor receptors (VEGFRs), playing a role in angiogenesis that, in turn, is detrimental in cancer progression (Deuquet et al., 2011). CMG2 is involved in the regulation cytoskeleton structure and might have a role in cancer spreading (Cryan and Rogers, 2011). The physiological functions of ANTXRs suggest that drugs targeting them would have a therapeutic potential for diseases where angiogenesis is detrimental (i.e., cancer and retinal neovascular diseases) (Cryan and Rogers, 2011).

The computational approach hereby presented aims at characterizing the homomeric and heteromeric interactions of PA. A PCN method was applied to crystal structures of the aforementioned complexes and was successful in finding "hot-spot" residues.

Analysis was focused on both global network stability (e.g., graph energy, flexibility, and robustness) and local features (e.g., participation coefficient, centrality, and degree). PCN approach has been further applied to evaluate complex stability, inferred from network resilience, or vulnerability (Oliva et al., 2013). The participation coefficient $P$ was the topological parameter that mostly affected the PCNs' vulnerability of all structures; meaning that, residues important for the protein-protein interactions are also involved in the inter-module communication. Inter-module communication is crucial for either allosteric mechanisms or cooperative events, which in turn play a key role in supramolecular interactions (Keskin et al., 2005). Therefore, identification of high $P$ residues will help the rational drug design of molecules targeting supramolecular (protein-protein) interactions of anthrax complexes.

\section{MATERIALS AND METHODS}

\section{Protein Data Set}

A series of X-ray structures were analyzed (Table 1). Structures are indexed with their own Protein Data Bank (PDB) code. The data set included monomeric and multimeric forms of PA, PA bound to $\mathrm{LF}$ (PDB: $3 \mathrm{KWV}$ ), and to human receptor CMG2 (PDB: 1T6B). 


\section{Protein Contact Network}

In PCN methodology, the protein structure is considered as a graph $\mathcal{G}=\{V, \varepsilon\}$, where the set $V$ includes the nodes $v_{1} \ldots, v_{\mathrm{n}}$ (i.e., the amino acid residues) and $\varepsilon$ is the set of links $\left(v_{\mathrm{i}}, v_{\mathrm{j}}\right)$, the link describes a specific relationship between $\mathrm{i}$-th and $\mathrm{j}$-th nodes. Topological descriptors of a PCN quantitatively describe the network that corresponds to a given protein structure (Csermely et al., 2013).

Protein contact networks were built on the base of three dimensional coordinates of $\alpha$-carbons exported from PDB files (Table 1). Nodes of the network correspond to residues connected by links if the Euclidean distance between $\alpha$-carbons is in the range of 4-8 $\AA$; this range has been chosen because it includes only significant non-covalent intra-molecular interactions (da Silveira et al., 2009). PCN building is based only on the adjacency binary matrix $\mathbf{A}$, whose rows and columns list residues ordered on the base of the protein primary sequence. A generic element $A_{\mathrm{ij}}$ of the adjacency matrix $\mathbf{A}$ is set to 1 if $\mathrm{i}$-th and $\mathrm{j}$-th nodes are connected by a link, otherwise $A_{\mathrm{ij}}$ is set to 0 .

Protein structures, translated into a PCN, were then described in terms of (Csermely et al., 2013) (Table 2):

- local network descriptors that describe single residues properties (Table 2, upper part);

- global network descriptors that correspond to properties of the whole structure (average on the whole graph) (Table 2, lower part).

Additionally, we computed two metrics of centrality:

- betweenness centrality: the number of shortest paths passing through each node. Nodes characterized by high betweenness centrality are crucial for signal transmission and, if placed in the PPI, likely responsible for inter-chain communication;

- closeness centrality: the sum of distances (shortest paths) of each node from other nodes. Indeed, high closeness centrality nodes are connected through few links to any other node of the network.

Furthermore, we applied a spectral clustering procedure in order to divide networks into clusters of nodes (Tasdighian et al., 2014). This procedure is based on eigenvalue decomposition. First of all, Laplacian matrix $\mathbf{L}$ of PCN is defined as the difference between the adjacency matrix $\mathbf{A}$ and the degree matrix $\mathbf{D}$, which is a diagonal matrix, whose generic element $D_{\text {ii }}$ is the $\mathrm{i}$-th node degree. The eigenvalue decomposition is applied to $\mathbf{L}$; therefore, eigenvectors are ordered according to the descending order of the

TABLE 1 | Protein data set.

\begin{tabular}{lll}
\hline PDB code & Description & Reference \\
\hline 1ACC & Anthrax toxin protective antigen (PA) & Petosa et al. (1997) \\
3Q8A & Anthrax PA wild type $(\mathrm{pH} \mathrm{5.5)}$ & Rajapaksha et al. (2012) \\
3Q8B & Anthrax PA wild type $(\mathrm{pH} \mathrm{9.0)}$ & Rajapaksha et al. (2012) \\
3KWV & Anthrax PA-PA-lethal factor & Feld et al. (2010) \\
3TEW & Crystal structure of PA & Feld et al. (2012) \\
1T6B & PA-CMG2 & Santelli et al. (2004) \\
3HVD & PA octamer & Kintzer et al. (2009)
\end{tabular}

corresponding eigenvalue. The reference for the spectral clustering decomposition is the second minor eigenvalue $\mathbf{v}_{\mathbf{2}}$. The whole range of $\mathbf{v}_{\mathbf{2}}$ values is divided into as many sub-ranges as the number of clusters (given as input); therefore, a node is placed in a cluster according to which sub-range its $\mathbf{v}_{\mathbf{2}}$ value belongs.

The relevance of this approach, to study the structural basis of allosteric regions, was previously reported (De Ruvo et al., 2012; Di Paola and Giuliani, 2015). Once the PCN is partitioned into clusters, each node (residue) can be characterized in terms of its propensity to form inter-cluster or intra-cluster connections, by means of two descriptors:

- the participation coefficient $P$, defined as:

$$
P_{\mathrm{i}}=1-\left(\frac{k_{\mathrm{si}}}{k_{\mathrm{i}}}\right)^{2}
$$

$k_{\mathrm{si}}$ is the node degree in its own cluster. $P$ measures the intercluster connectivity of nodes. $P$ has been found to shift from non-null to null values in regions close to an allosteric site (De Ruvo et al., 2012);

- $z$-score of intra-cluster connectivity, defined as:

$$
z_{\mathrm{i}}=\frac{k_{\mathrm{i}}-\bar{k}_{\mathrm{si}}}{\sigma_{\mathrm{si}}}
$$

$\bar{k}_{\mathrm{si}}$ and $\sigma_{\mathrm{si}}$ are, respectively, the average and the SD of degree in the cluster to which the i-th node belongs. Therefore, $z$-score

TABLE 2 | Topological descriptors

\begin{tabular}{ll}
\hline Variable & Description \\
\hline LOCAL & $\begin{array}{l}\text { Node degree } k_{\mathrm{i}} \text { is defined as the number of links each node } \\
\text { forms: }\end{array}$ \\
$\qquad k_{\mathrm{i}}=\sum_{\mathrm{j}} A_{\mathrm{ij}}$
\end{tabular}

$\begin{array}{ll}\text { Clustering } & \text { The clustering coefficient } C_{\mathrm{i}} \text { computes the number of } \\ \text { coefficient } & \text { triangles in a network where a node is involved }\end{array}$

Contact order Contact order ord computes for each node the average range (distance in sequence of adjacent nodes) of its contacts (Oliva et al., 2013):

$$
\operatorname{ord}_{i}=\frac{\sum_{j \neq i}|i-j| A_{i j}}{k_{i}}
$$

\section{GLOBAL}

Average degree The average degree $\bar{k}$ is the average value of node degree $k_{i}$

Average clustering The average clustering coefficient $\bar{C}$ is the average value of coefficient node clustering coefficient $C_{i}$

Average shortest The average shortest path asp is the average value of the path shortest paths matrix $S P$ over all pair of residues

Graph energy The graph energy $E$ is defined as the sum of the adjacency matrix eigenvalues in module

Graph energy of The graph energy of complex formation $E_{C}$ is computed as complexes difference between the graph energy $E$ computed for the whole complex and the corresponding value for single interacting chains

Inter-chain number The inter-chain number of contacts $/ C$ represents the number of contacts of residues in pairwise contacts between different chains

Local (single residue) and global (whole structure) descriptors of the topology of protein contact networks. 
describes the propensity of a node to establish links with nodes belonging to its own cluster.

According to $P$ and $z$ values, nodes can be classified into seven categories, which cover specific regions in the $P-z$ plane. This representation, called Guimerà-Amaral cartography (Guimerà and Nunes Amaral, 2005), characterizes the topological role of nodes in the PCN, according to their function in signal transmission across the network (Cumbo et al., 2014). The methodology is based on an evolution of the degree concept, which accounts for the ability of the nodes to link different regions of the network. This perspective is particularly well suited for PCNs, which are characterized by a strong small-world character; average shortest path in small-world networks scales logarithmically with the total number of vertices due to long-range contacts between residues far in sequence (Watts and Strogatz, 1998; Atilgan et al., 2004; Paci et al., 2012). $P-z$ maps (representation of nodes distribution on the $P-z$ planes) show a peculiar shape, referred to as "dentist's chair," which represents the PCNs of the folded protein structures. The "dentist's chair" shape is conserved for a large number of structures, and reveals a common architecture of protein folds regardless of protein function and size (Krishnan et al., 2007). Clustering partition, through a clustering color map, was hereby represented as a 2D map clusters of residues (Tasdighian et al., 2014), where each cluster is represented by a different color and projections of clusters correspond to long-range interactions. This representation helps in characterizing the match between clusters and chains, or protein domains.

\section{Flexibility and Vulnerability Assessment}

The flexibility index $f$ of a network with $c$ degrees of freedom is defined as (Thorpe and Kuhn, 2000):

$$
f=\frac{c-6}{c}
$$

because the maximum number of degrees of freedom in a rigid network in $3 \mathrm{D}$ is 6 .

The $f$ index was calculated accordingly to Eq. 3 (Table 3 ) and degrees of freedom of PCNs were calculated accordingly to the method of Zelazo et al. (2012). The vulnerability of PCNs was computed in terms of size reduction of the largest giant component (LGC) of each PCN (Holme et al., 2002; Oliva et al., 2013); this reduction of size is defined also as "biggest fall." The LGC of the graph is the set of connected nodes with the maximum number of nodes. The reduction profile of the size of LGC depends on the specific strategy of nodes removal: nodes with decreasing

TABLE 3 | Flexibility analysis results.

\begin{tabular}{lcc}
\hline PDB code & Degrees of freedom & Flexibility $\boldsymbol{f}$ \\
\hline 1ACC & 36 & 0.83 \\
3Q8A & 40 & 0.85 \\
3Q8B & 38 & 0.84 \\
3TEW & 46 & 0.87 \\
3KWV & 30 & 0.80 \\
3HVD & 21 & 0.71 \\
1T6B & 26 & 0.77
\end{tabular}

or increasing values of a given network descriptor are removed. A huge fall in LGC size, due to a small fraction of removed nodes, identifies a network vulnerable to a given removal strategy, whereas the network is robust if a smooth and regular degradation is measured.

The synthetic index of vulnerability, the Degradation Index DI, (Oliva et al., 2013), is defined as the normalized metric of the effectiveness of different removal strategies:

$$
\mathrm{DI}=\frac{\text { Difference in giant component size }}{\text { Fraction of removed nodes }}
$$

The index is computed at the first discontinuity in size of the giant component and accounts for the magnitude of the fall of the LGC and for the fraction of removed nodes that generated the fall.

\section{Figures, graphs, and statistics}

Figures of the protein structures were obtained via PyMOL Molecular Graphics System, Version 1.7.4. Values of the molecular descriptors of residues were mapped in each structure by adding the column corresponding to the $b$-factor in the PDB file. Graphs and statistics were obtained with Matlab 2014a environment.

\section{RESULTS}

\section{Protein Contact Networks General Properties}

Global topological descriptors of PCNs (Table 4) of monomeric forms were not markedly different from descriptors of complexes; this result suggested that PPIs would not influence the overall topology of PCNs. The parameter that best described PPIs is the inter-chain energy of the graph $E_{\mathrm{C}}$. The percentage of residues involved in inter-chain contacts (IC) varied from 8.7\% (PDB: $3 \mathrm{KWV}$ ) to $4.4 \%$ (PDB: $1 \mathrm{~T} 6 \mathrm{~B}$ ), whereas the inter-chain energy $E_{\mathrm{C}}$ normalized per inter-chain contacts $\left(E_{\mathrm{C}} / I C\right)$ substantially did not vary; meaning that $E_{\mathrm{C}}$ only depends on the number of residues in the PPIs and not on their nature (contact length and type). Greater $E_{C}$ values described complexes bearing homomeric PA interactions (PDB: $3 \mathrm{HVD}$ and $3 \mathrm{KWV}$ ).

\section{"Hot-Spots" Recognition by Mesoscopic Topological Descriptors}

Protective agent bound to LF (PDB: $3 \mathrm{KWV}$ ) and to receptor CMG2 (PDB: 1T6B) was analyzed (Figure 1), because drugs targeting key residues of such complexes may be developed as anthrax antidotes or novel anti-angiogenic molecules.

The clustering of PCN of $3 \mathrm{KWV}$, accordingly to graph-spectral method, was carried out (Figure 2). The protein network was divided into three clusters. The clustering helped for the identification of protein modules of the complex. The two chains of PA were not recognized as two different clusters; otherwise, each PA chain was divided into two functional modules: the red cluster (Figure 2A) is the protein module far from PPI (red cartoon Figure 2B), the yellow cluster (Figure 2A) is the protein module near the PPI (yellow cartoon Figure 2B) and in contact to the LF (green cartoon Figure 2B). The red and yellow clusters were substantially intermingled (Figure 2A). 
TABLE 4 | Topological and structural descriptors.

\begin{tabular}{|c|c|c|c|c|c|c|c|c|c|c|c|c|}
\hline & $N$ & $\bar{k}$ & $\bar{c}$ & $\overline{\mathbf{S P}}$ & $E$ & ORD & $\mathrm{E}_{\mathrm{C}}$ & IC (\%) & $E_{\mathrm{c}} / I C$ & $\boldsymbol{R}_{\mathrm{G}}$ & MDF & $\varepsilon$ \\
\hline $1 \mathrm{ACC}$ & 665 & 7.91 & 0.30 & 7.77 & 1462.1 & 302.18 & - & - & - & 16.11 & 1.89 & 0.68 \\
\hline 3Q8A & 675 & 7.87 & 0.30 & 7.81 & 1476.8 & 288.91 & - & - & - & 16.09 & 1.90 & 0.64 \\
\hline 3Q8B & 676 & 7.95 & 0.30 & 7.77 & 1486 & 298.71 & - & - & - & 16.12 & 1.86 & 0.64 \\
\hline 3TEW & 717 & 8.03 & 0.29 & 7.90 & 1570.8 & 306.17 & - & - & - & 16.42 & 1,56 & 0.63 \\
\hline $3 \mathrm{KWV}$ & 1275 & 7.71 & 0.29 & 9.86 & 2767.8 & 337.64 & 38.45 & $111(8.7)$ & 0.35 & 18.89 & 1.80 & 0.75 \\
\hline $3 \mathrm{HVD}$ & 1041 & 7.98 & 0.30 & 8.73 & 2305.7 & 349.41 & 23.4 & 69 (6.6) & 0.34 & 16.25 & 2.40 & 0.73 \\
\hline $1 \mathrm{~T} 6 \mathrm{~B}$ & 846 & 8.08 & 0.29 & 9.10 & 1876.2 & 304.52 & 14.33 & $37(4.4)$ & 0.39 & 19.25 & 0.87 & 0.78 \\
\hline
\end{tabular}

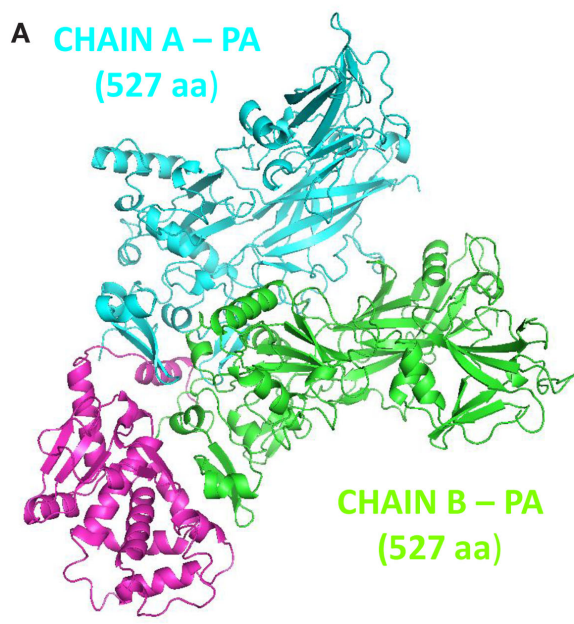

CHAIN C - LF

(221 aa)

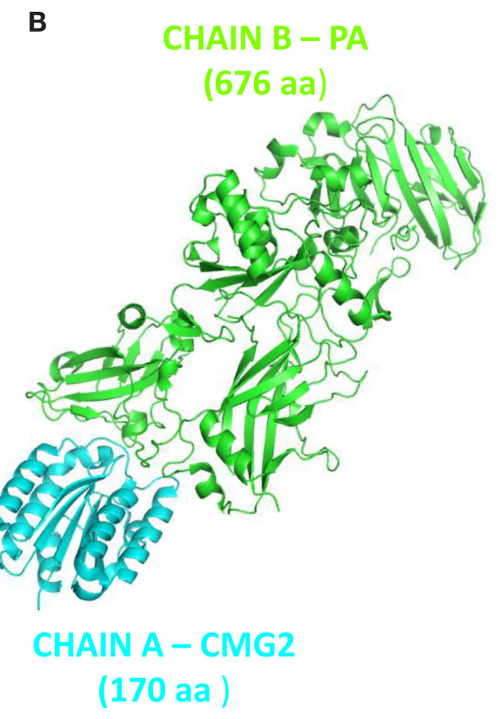

(170 aa)

FIGURE 1 | Complex structures: (A) PA-PA-LF trimeric complex (PDB: 3KWV); (B) dimeric complex PA-CMG2 (PDB: 1T6B).
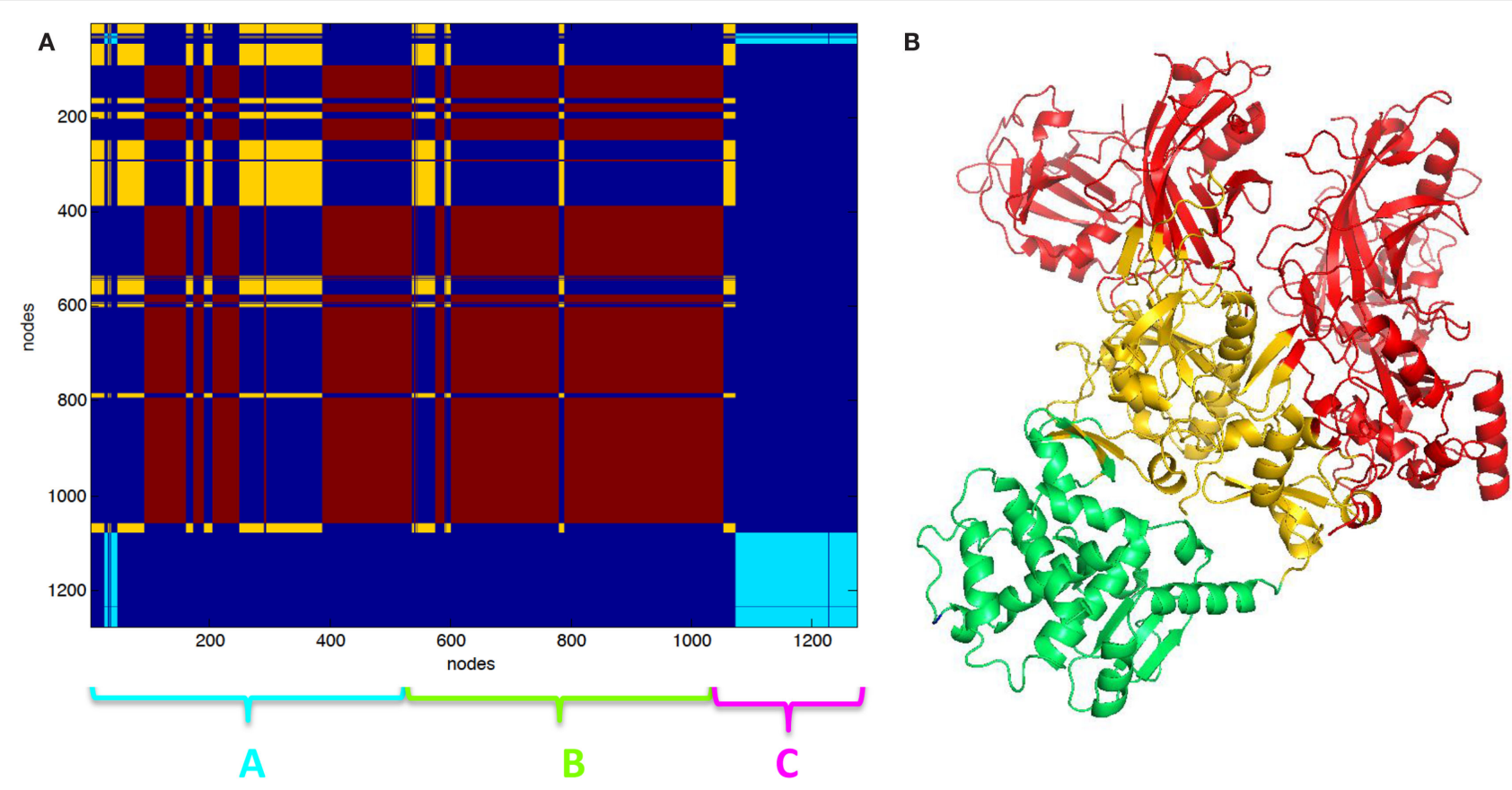

FIGURE 2 | Clustering partition of PA-PA-LF complex: (A) clustering color map, the length of the three chains is reported for reference; (B) cartoon representation of clusters the green cartoon corresponds to the light blue cluster in (A). The lethal factor (LF) is highlighted by the empty circle. 

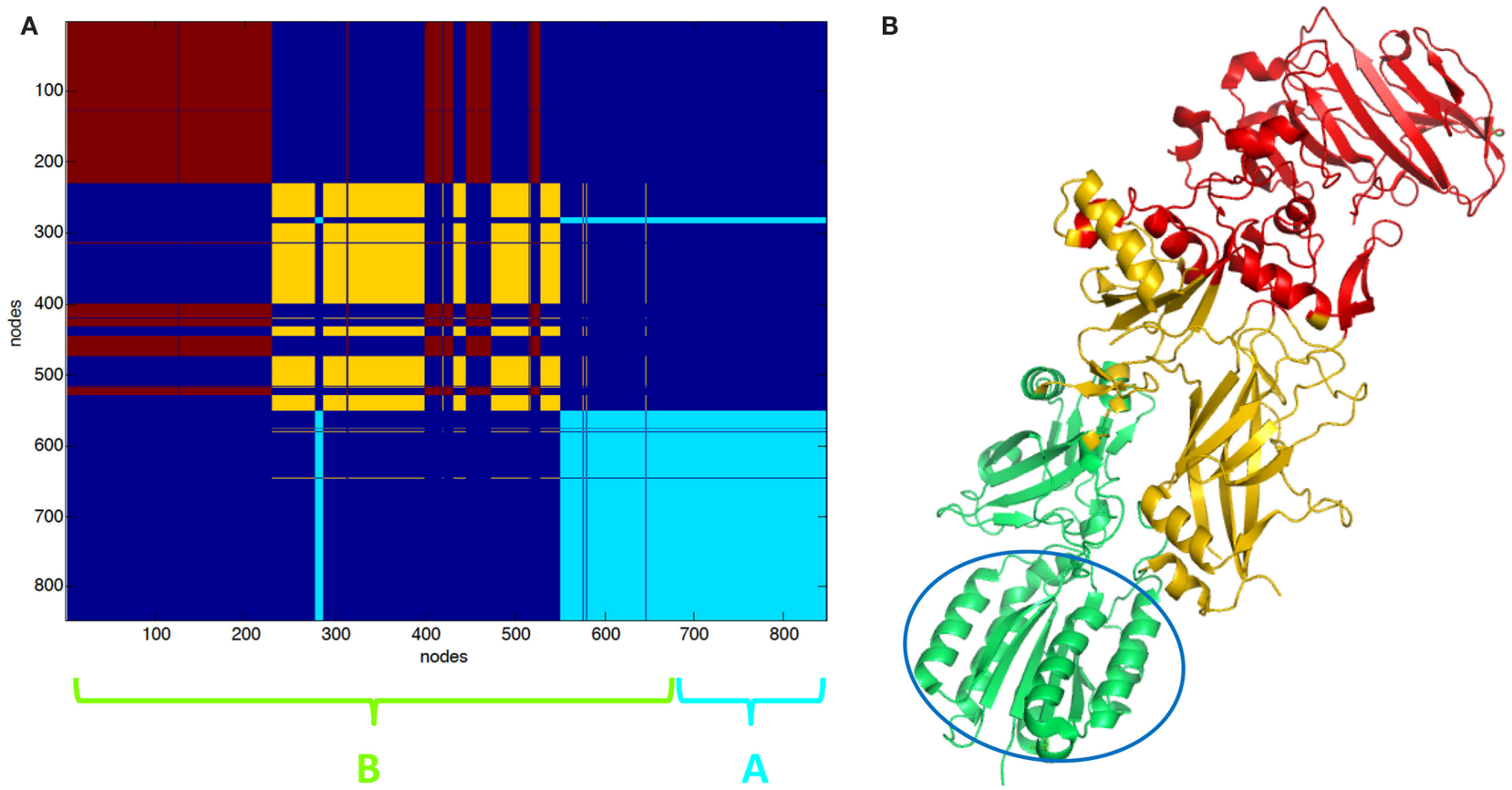

FIGURE 3 | Clustering partition of PA-CMG2 complex: (A) clustering color map, the length of the three chains is reported for reference; (B) cartoon representation of clusters the green cartoon corresponds to the light blue cluster in (A). The CMG2 receptor is highlighted by the empty circle.
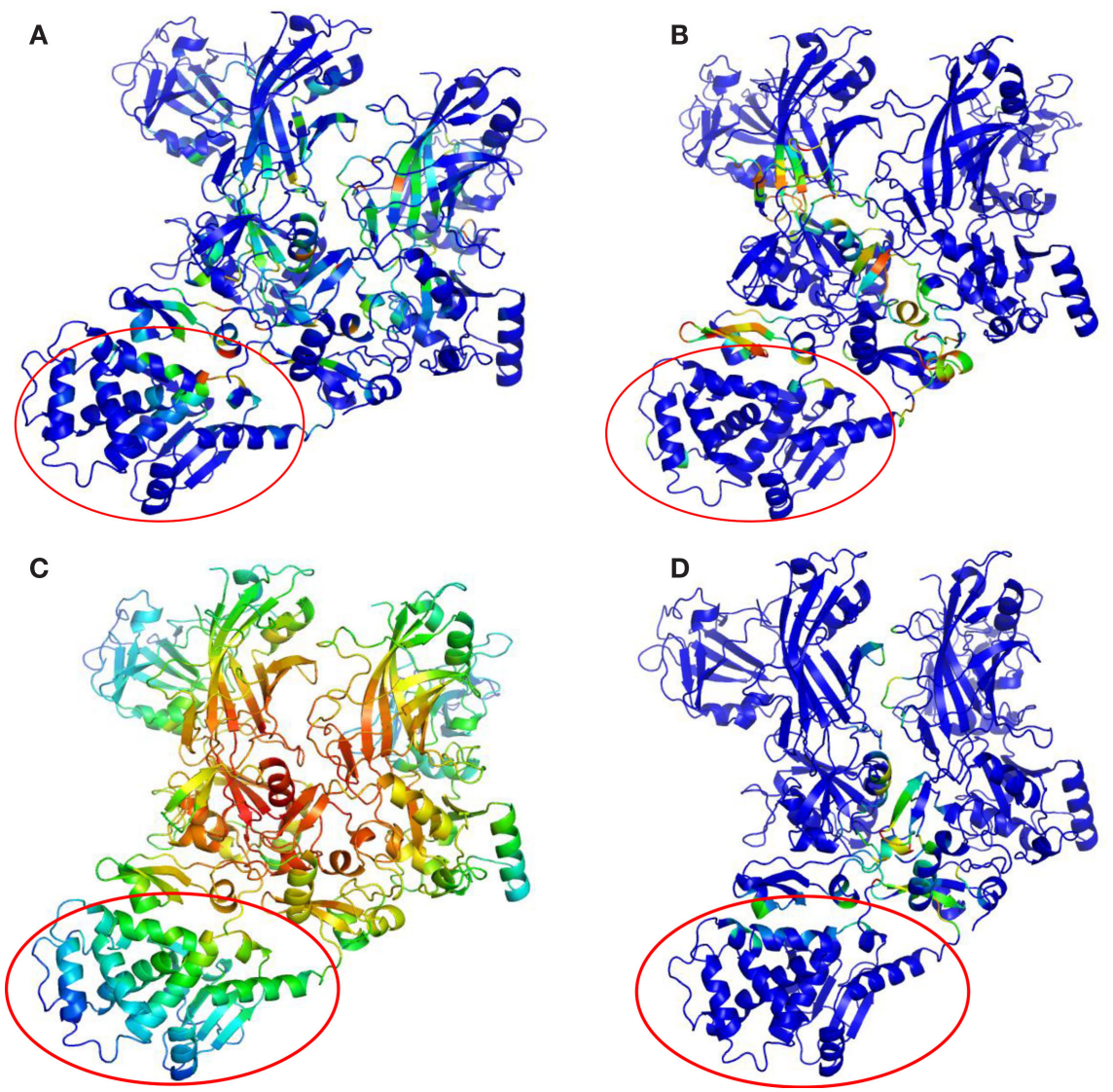

FIGURE 4 | Maps of PCN descriptors on protein structures: complex PA-PA-LF (PDB: 3KWV): (A) betweenness centrality; (B) participation coefficient $P$; (C) closeness centrality; (D) inter-chain degree. The lethal factor (LF) is highlighted by the empty circle. 
Partition in three clusters of PA-CMG2 complex (1T6B) was also carried out. In 1T6B, a single PA chain is bound to CMG2. $\mathrm{PA}$ is divided into two intermingled functional domains, such as the yellow and the red clusters (Figures 3A,B). The light blue (Figure 3A) cluster includes the CGM2 and a domain of PA (cartoon Figure 3B).

Key PCN descriptors were mapped in the molecular representation of PA-PA-LF (PDB: 3KWV) and PA-CGM2 (PDB: 1T6B) (Figures 4 and 5). In $3 \mathrm{KWV}$, the residues with highest closeness centrality (Figures $4 \mathrm{C}$ and $5 \mathrm{C}$ ) belong to the center of mass of the complex, where the residues have the lowest flexibility. In fact, high closeness corresponds to high rigidity of the residue. Conversely, betweenness centrality (Figures $\mathbf{4 A}$ and $5 \mathbf{A}$ ) and participation coefficient $P$ (Figures $4 B$ and 5B) identify residues at the PPIs, which would be good candidates as hot-spot residues. Betweenness centrality and participation coefficient $P$ maps also highlighted residues far from interfaces, in regions with possible allosteric function. Finally, the inter-chain degree (Figure 4D) identified the residues in PPIs easily. The role of inter-chain degree in the identification of residues at PPIs (PA-LF, PA-PA, and PA-CMG2) confirmed the results about the role of $E_{\mathrm{c}}$ in description of PPIs of complexes.

\section{Protein Contact Networks Rigidity and Vulnerability Analysis}

All structures showed a flexibility index $f$ (Table 3) $>0.7$, albeit with some differences. 3TEW was the most flexible $(f=0.8696)$, and 3 HVD was the most rigid $(f=0.7143)$. Flexibility was high in monomeric forms of PA, and decreased with increasing number of interacting chains. In fact, flexibility was lowest in 3HVD where eight chains of PA are interacting. The comparison of flexibility of PA-PA-LF (PDB: 3KWV) and PA-CMG2 (PDB: 1T6B) revealed that the number of PA chains did not decrease the flexibility of $3 \mathrm{KWV}$; although, the difference in values was not high.

Vulnerability analysis was done by removing nodes with decreasing values of $P$, contact order, and degree (Table 5; Figures 6 and 7). The $P$ removal strategy significantly influenced the dimension of LGC, i.e., the biggest fall of LGC is associated to the lowest percentage of removed nodes. In all structures, the
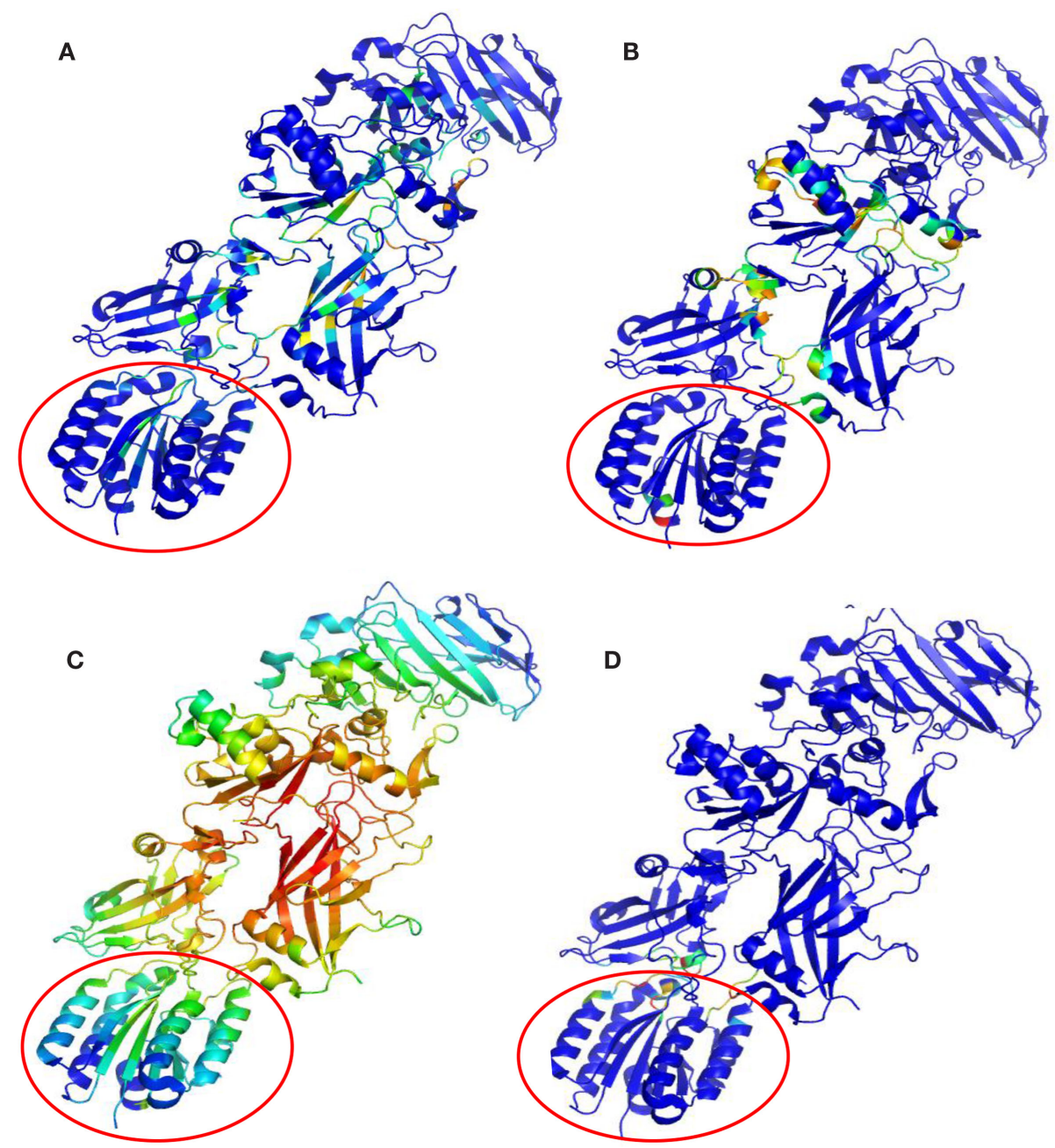

FIGURE 5 | Maps of PCN descriptors on protein structures: complex PA-CGM2 (PDB: 1T6B): (A) betweenness centrality; (B) participation coefficient $P$; (C) closeness centrality; (D) inter-chain degree. The CMG2 receptor is highlighted by the empty circle. 
participation coefficient $P$ was the parameter with the highest influence on PCN robustness. The most vulnerable structure was the octameric PA (PDB: 3HVD), which showed the biggest fall with $3 \%$ of removed nodes. The PA-PA-LF complex was less vulnerable than PA-CGM2; meaning that PA-PA interaction were not crucial in the participation coefficient $P$ vulnerability strategy, at least for heteromeric interactions. The contact order removal strategy was substantially detrimental for network robustness of the octameric structure of PA (PDB: 3HVD) (7\% of removed nodes) and of PA-PA-LF complex (PDB: 3KWV) (16\% of removed nodes); in this case, $\mathrm{PA}-\mathrm{PA}$ interaction influenced the robustness of PCNs. Finally, the degree removal strategy did not influence the robustness of all PCNs. The influence of $P$ parameter was different in homomeric (PDB: 3HVD) and heteromeric complexes (PDB: $3 \mathrm{KWV}$ ). Map of residues with high $P$ values in the

TABLE 5 | Vulnerability analysis

\begin{tabular}{|c|c|c|c|}
\hline PDB code & Biggest fall & \% Removed nodes & DI index \\
\hline \multicolumn{4}{|c|}{ DESCENDING P } \\
\hline $1 \mathrm{ACC}$ & 286 (43.01\%) & 9.62 & 4.47 \\
\hline 3Q8A & 301 (44.59\%) & 9.93 & 4.49 \\
\hline 3Q8B & $287(42.46 \%)$ & 10.21 & 4.16 \\
\hline 3TEW & $306(42.68 \%)$ & 6.28 & 6.8 \\
\hline $3 \mathrm{KWV}$ & $151(28.65 \%)$ & 9.68 & 2.96 \\
\hline 3HVD & $482(46.30 \%)$ & 3.17 & 14.61 \\
\hline $1 \mathrm{~T} 6 \mathrm{~B}$ & 363 (42.91\%) & 6.38 & 6.72 \\
\hline \multicolumn{4}{|c|}{ DESCENDING CONTACT ORDER } \\
\hline $1 \mathrm{ACC}$ & 203 (30.53\%) & 23.16 & 1.32 \\
\hline 3Q8A & $184(27.26 \%)$ & 21.33 & 1.28 \\
\hline 3Q8B & $210(31.07 \%)$ & 20.12 & 1.54 \\
\hline 3TEW & $196(27.34 \%)$ & 20.78 & 1.32 \\
\hline $3 \mathrm{KWV}$ & $219(41.56 \%)$ & 16.13 & 2.58 \\
\hline 3HVD & $481(46.21 \%)$ & 7.20 & 6.41 \\
\hline $1 \mathrm{~T} 6 \mathrm{~B}$ & $310(36.64 \%)$ & 21.16 & 1.73 \\
\hline \multicolumn{4}{|c|}{ DESCENDING DEGREE } \\
\hline $1 \mathrm{ACC}$ & $114(17.14 \%)$ & 42.86 & 0.40 \\
\hline 3Q8A & $130(19.26 \%)$ & 41.93 & 0.46 \\
\hline 3Q8B & $144(21.30 \%)$ & 41.12 & 0.52 \\
\hline 3TEW & 169 (23.57\%) & 41.98 & 0.56 \\
\hline $3 \mathrm{KWV}$ & $64(12.14 \%)$ & 44.21 & 0.27 \\
\hline $3 H V D$ & 245 (23.54\%) & 43.90 & 0.54 \\
\hline $1 \mathrm{~T} 6 \mathrm{~B}$ & $100(11.82 \%)$ & 48.94 & 0.244 \\
\hline
\end{tabular}

homomeric (Figure 7A) and heteromeric (Figure 7B) complexes of PA is reported in Figure 7. High $P$ residues were at PA-PA interfaces; however, high $P$ values in $3 \mathrm{KWV}$ were localized also at PA-LF interface.

\section{DISCUSSION}

Crystal structures of unbound anthrax PA, octameric form of PA, PA-PA-LF, and PA-CMG2 complexes were analyzed using a PCN approach. This methodology was used in order to identify residues (hot-spots) that influence significantly the stability of complexes.

Protein-protein interface properties were largely independent from global topology descriptor of the systems. For this reason, local (single residue) properties of PCNs were analyzed in detail.

Local topological descriptors that better described PPIs were the betweenness centrality, the participation coefficient $P$, and the inter-chain degree. Furthermore, hot-spot residues with the highest betweenness centrality and the participation coefficient were localized in regions with allosteric functions. This result complies with previous works, hot-spot residues are not necessarily placed PPIs, and they can be localized in other regions of the complex acting through allosteric mechanisms (Reynolds et al., 2011). Residues far from PPIs (Reynolds et al., 2011) modulate the smallworld properties of PCNs explaining the allosteric nature of PPIs (Brinda et al., 2002).

Thus, the general wiring architecture of $\mathrm{PCNs}$ provides a mesoscopic framework (clustering, small-world nature) influencing the "hot-spot" character of residues. This result is in accordance with the previous findings about the modular feature of protein structures (Di Paola et al., 2012; Tasdighian et al., 2014).

Analysis of global PCN vulnerability identified which residues have a crucial role in the stability of anthrax complexes. The PCN vulnerability assessment showed that high $P$ residues played a key role in the network stability of all analyzed protein structures. Furthermore, participation coefficient $P$ and the contact order described residues likely to be involved in cooperative binding. In the PA octamer (3HVD), the most vulnerable structure, high $P$ residues were mostly localized at PPIs; this means that intermodule communication guide cooperative binding and influence
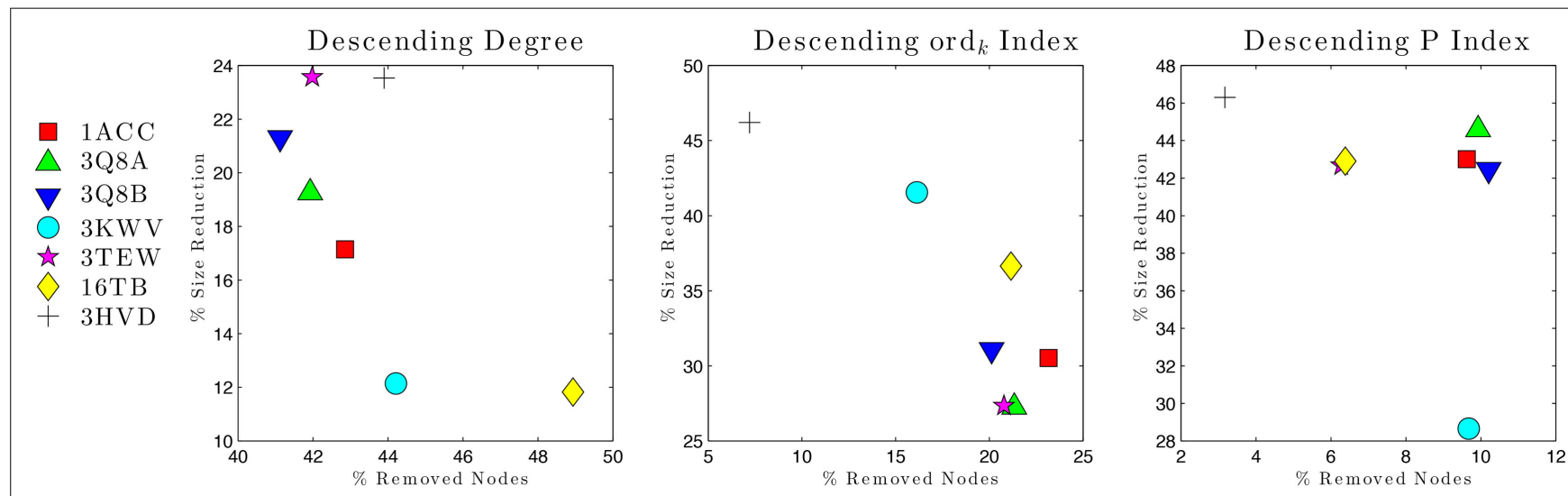

FIGURE 6 | Network vulnerability upon nodes removal strategies. 


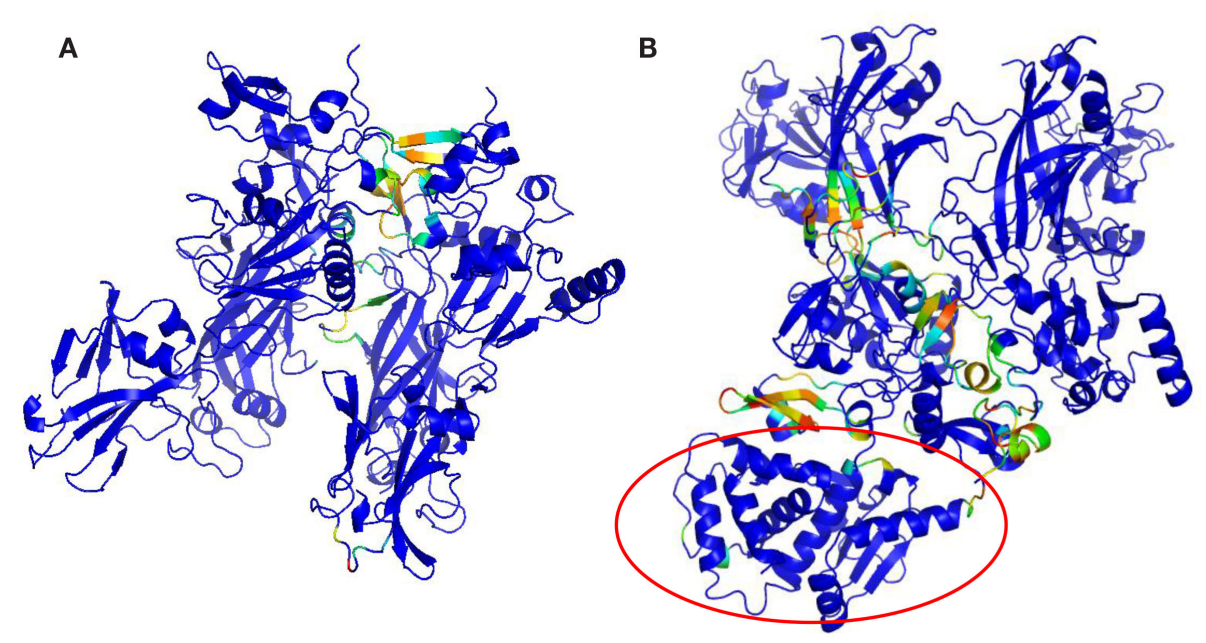

FIGURE 7 |P maps on protein structures: (A) 3HVD, octamer of PA; (B) 3KWV, PA-PA-LF complex.

PCN robustness. In the PA-PA-LF complex, high $P$ residues were specifically at PA-LF and PA-PA interfaces and also in allosteric regions. Furthermore, PA-CMG2 complex was more vulnerable than PA-PA-LF based on $\mathrm{P}$ removal strategy. Thus, the participation coefficient $P$ accounted for overall structural stability and high $P$ residues were localized at PPIs and in allosteric regions. Furthermore, the participation coefficient $P$ partially explained cooperative events. However, the parameter that mostly explained cooperative events was the contact order, because the most vulnerable structures were the octameric PA and the PA-PA-LF complexes, where there are homomeric PA-PA interactions. Interestingly, all analyzed structures were similarly resistant to node removal based on decreasing degree, which is known to be highly effective for scale-free networks (Holme et al., 2002). In conclusion, network vulnerability assessment identified high $P$ residues, distributed both at PPIs and regions far from PPIs, as the most essential for global stability.

The identification of high $P$ residues, crucial for supramolecular interactions, would have a perspective application as a guide

\section{REFERENCES}

Arkin, M. R., Tang, Y., and Wells, J. A. (2014). Small-molecule inhibitors of proteinprotein interactions: progressing toward the reality. Chem. Biol. 21, 1102-1114. doi:10.1016/j.chembiol.2014.09.001

Atilgan, A. R., Akan, P., and Baysal, C. (2004). Small-world communication of residues and significance for protein dynamics. Biophys. J. 86(1 Pt 1), 85-91. doi:10.1016/S0006-3495(04)74086-2

Brinda, K. V., Kannan, N., and Vishveshwara, S. (2002). Analysis of homodimeric protein interfaces by graph-spectral methods. Protein Eng. 15, 265-277. doi:10. 1093/protein/15.4.265

Brinda, K. V., and Vishveshwara, S. (2005). Oligomeric protein structure networks: insights into protein-protein interactions. BMC Bioinformatics 6:296. doi:10. 1186/1471-2105-6-296

Cheng, S., Fu, H.-L., and Cui, D.-X. (2015). Characteristics analyses and comparisons of the protein structure networks constructed by different methods. Interdiscip. Sci. 1-10. doi:10.1007/s12539-015-0106-y

Chennubhotla, C., Rader, A., Yang, L.-W., and Bahar, I. (2005). Elastic network models for understanding biomolecular machinery: from enzymes to for site-directed mutagenesis and other experimental approaches, aimed at functional characterization of PPIs. Furthermore, this approach will help medicinal chemists to identify cavities for molecular-docking studies, in order to design effective drugs targeting protein-protein interactions. Indeed, finding of high $P$ residues will open additional scenarios to drug discovery applications. Drugs may not target PPIs, often scarcely accessible. Additionally, drugs would be designed to bind allosteric regions, influencing supramolecular interactions with efficacy comparable or higher than drugs targeting only protein-protein interfaces.

\section{ACKNOWLEDGMENTS}

The authors wish to thank the reviewers and the editor for their comments, which contributed to improve the manuscript considerably. Dr. CP would like to thank Prof. Filippo Drago, chair of the Department of Biomedical and Biotechnological Sciences (BIOMETEC) of University of Catania, where part of the work on this paper was done.

supramolecular assemblies. Phys. Biol. 2, S173-S180. doi:10.1088/1478-3975/2/ 4/S12

Clackson, T., and Wells, J. (1995). A hot spot of binding energy in a hormone-receptor interface. Science 267, 383-386. doi:10.1126/science. 7529940

Cryan, L. M., and Rogers, M. S. (2011). Targeting the anthrax receptors, TEM8 and CMG-2, for anti-angiogenic therapy. Front. Biosci. (Landmark Ed.) 16:1574-1588. doi:10.2741/3806

Csermely, P., Korcsmáros, T., Kiss, H. J., London, G., and Nussinov, R. (2013). Structure and dynamics of molecular networks: a novel paradigm of drug discovery: a comprehensive review. Pharmacol. Ther. 138, 333-408. doi:10.1016/ j.pharmthera.2013.01.016

Cumbo, F., Paci, P., Santoni, D., Di Paola, L., and Giuliani, A. (2014). GIANT: a cytoscape plugin for modular networks. PLoS ONE 9:e105001. doi:10.1371/ journal.pone.0105001

da Silveira, C. H., Pires, D. E. V., Minardi, R. C., Ribeiro, C., Veloso, C. J. M., Lopes, J. C. D., et al. (2009). Protein cutoff scanning: a comparative analysis of cutoff dependent and cutoff free methods for prospecting contacts in proteins. Proteins 74, 727-743. doi:10.1002/prot.22187 
De Ruvo, M., Giuliani, A., Paci, P., and Santoni, D. (2012). Shedding light on protein-ligand binding by graph theory: the topological nature of allostery. Biophys. Chem. 16, 21-29. doi:10.1016/j.bpc.2012.03.001

Deuquet, J., Lausch, E., Superti-Furga, A., and van der Goot, F. G. (2011). The dark sides of capillary morphogenesis gene 2. EMBO J. 31, 3-13. doi:10.1038/emboj. 2011.442

Di Paola, L., De Ruvo, M., Paci, P., Santoni, D., and Giuliani, A. (2013). Protein contact networks: an emerging paradigm in chemistry. Chem. Rev. 113, 1598-1613. doi: $10.1021 / \mathrm{cr} 3002356$

Di Paola, L., and Giuliani, A. (2015). Protein contact network topology: a natural language for allostery. Curr. Opin. Struct. Biol. 31, 43-48. doi:10.1016/j.sbi.2015. 03.001

Di Paola, L., Mei, G., Di Venere, A., and Giuliani, A. (2015). Exploring the stability of dimers through protein structure topology. Curr. Protein Pept. Sci.

Di Paola, L., Paci, P., Santoni, D., De Ruvo, M., and Giuliani, A. (2012). Proteins as sponges: a statistical journey along protein structure organization principles. $J$. Chem. Inf. Model. 52, 474-482. doi:10.1021/ci2005127

Feld, G. K., Kintzer, A. F., Tang, I. I., Thoren, K. L., and Krantz, B. A. (2012). Domain flexibility modulates the heterogeneous assembly mechanism of anthrax toxin protective antigen. J. Mol. Biol. 415, 159-174. doi:10.1016/j.jmb. 2011.10.035

Feld, G. K., Thoren, K. L., Kintzer, A. F., Sterling, H. J., Tang, I. I., Greenberg, S. G., et al. (2010). Structural basis for the unfolding of anthrax lethal factor by protective antigen oligomers. Nat. Struct. Mol. Biol. 17, 1383-1390. doi:10.1038/ nsmb.1923

Fu, S., Tong, X., Cai, C., Zhao, Y., Wu, Y., Li, Y., et al. (2010). The structure of tumor endothelial Marker 8 (TEM8) extracellular domain and implications for its receptor function for recognizing anthrax toxin. PLoS ONE 5:e11203. doi:10.1371/journal.pone.0011203

Goel, A. K. (2015). Anthrax: a disease of biowarfare and public health importance. World J. Clin. Cases 3, 20. doi:10.12998/wjcc.v3.i1.20

Guimerà, R., and Nunes Amaral, L. A. (2005). Functional cartography of complex metabolic networks. Nature 433, 895-900. doi:10.1038/nature03288

Holme, P., Kim, B. J., Yoon, C. N., and Han, S. (2002). Attack vulnerability of complex networks. Phys. Rev. E Stat. Nonlin. Soft Matter Phys. 65, 056109. doi:10.1103/PhysRevE.65.056109

Jernigan, D. B., Raghunathan, P. L., Jernigan, D. B., Bell, B. P., Brechner, R., Raghunathan, P. L., et al. (2002). Investigation of bioterrorism-related anthrax, United States, 2001: epidemiologic findings. Emerging Infect. Dis. 8, 1019-1028. doi:10.3201/eid0810.020353

Jernigan, J. A., Stephens, D. S., Ashford, D. A., Omenaca, C., Topiel, M. S., Galbraith, M., et al. (2001). Bioterrorism-related inhalational anthrax: the first 10 cases reported in the United States. Emerging Infect. Dis. 7, 933-944. doi:10.3201/ eid0706.010604

Karain, W. I., and Qaraeen, N. I. (2015). Weighted protein residue networks based on joint recurrences between residues. BMC Bioinformatics 16:173. doi:10.1186/ s12859-015-0621-1

Keskin, O., Ma, B., Rogale, K., Gunasekaran, K., and Nussinov, R. (2005). Proteinprotein interactions: organization, cooperativity and mapping in a bottomup systems biology approach. Phys. Biol. 2, S24-S35. doi:10.1088/1478-3975/2/ $2 / \mathrm{S} 03$

Kintzer, A. F., Thoren, K. L., Sterling, H. J., Dong, K. C., Feld, G. K., Tang, I. I., et al. (2009). The protective antigen component of anthrax toxin forms functional octameric complexes. J. Mol. Biol. 392, 614-629. doi:10.1016/j.jmb. 2009.07.037

Krishnan, A., Giuliani, A., Zbilut, J. P., and Tomita, M. (2007). Network scaling invariants help to elucidate basic topological principles of proteins. J. Proteome Res. 6, 3924-3934. doi:10.1021/pr070162v

Morrow, J. K., and Zhang, S. (2012). Computational prediction of protein hot spot residues. Curr. Pharm. Des. 18, 1255-1265. doi:10.2174/138161212799436412
Oliva, G., Di Paola, L., Giuliani, A., Pascucci, F., and Setola, R. (2013). "Assessing protein resilience via a complex network approach," in IEEE 2nd International Workshop on Network Science. West Point, NY. 131-137. doi:10.1109/NSW. 2013.6609209

Paci, P., Di Paola, L., Santoni, D., De Ruvo, M., and Giuliani, A. (2012). Structural and functional analysis of hemoglobin and serum albumin through protein long-range interaction networks. Curr. Proteom. 9, 160-166. doi:10.2174/ 157016412803251815

Petosa, C., Collier, R. J., Klimpel, K. R., Leppla, S. H., and Liddington, R. C. (1997). Crystal structure of the anthrax toxin protective antigen. Nature 385, 833-838. doi:10.1038/385833a0

Plaxco, K. W., Simons, K. T., Ruczinski, I., and Baker, D. (2000). Topology, stability, sequence, and length: defining the determinants of two-state protein folding kinetics. Biochemistry 39, 11177-11183. doi:10.1021/bi000200n

Rajapaksha, M., Lovell, S., Janowiak, B. E., Andra, K. K., Battaile, K. P., and Bann, J. G. (2012). pH effects on binding between the anthrax protective antigen and the host cellular receptor CMG2. Protein Sci. 21, 1467-1480. doi:10.1002/pro.2136

Reynolds, K. A., McLaughlin, R. N., and Ranganathan, R. (2011). Hot spots for allosteric regulation on protein surfaces. Cell 147, 1564-1575. doi:10.1016/j.cell. 2011.10.049

Santelli, E., Bankston, L. A., Leppla, S. H., and Liddington, R. C. (2004). Crystal structure of a complex between anthrax toxin and its host cell receptor. Nature 430, 905-908. doi:10.1038/nature02763

Tasdighian, S., Di Paola, L., De Ruvo, M., Palumbo, P., Paci, P., Santoni, D., et al. (2014). Modules identification in protein structures: the topological and geometrical solutions. J. Chem. Inf. Model. 54, 159-168. doi:10.1021/ ci $400218 \mathrm{v}$

Thorn, K. S., and Bogan, A. A. (2001). ASEdb: a database of alanine mutations and their effects on the free energy of binding in protein interactions. Bioinformatics 17, 284-285. doi:10.1093/bioinformatics/17.3.284

Thorpe, M. F., and Kuhn, L. (2000). Rigidity in glasses and proteins. Periodica Mathematica Hungarica 39, 241-252. doi:10.1023/A:1004867612889

Watts, D. J., and Strogatz, S. H. (1998). Collective dynamics of "small-world" networks. Nature 393, 440-442. doi:10.1038/30918

Wein, L. M., Wein, L. M., Craft, D. L., Kaplan, E. H., Craft, D. L., and Kaplan, E. H. (2003). Emergency response to an anthrax attack. Proc. Natl. Acad. Sci. U.S.A. 100, 4346-4351. doi:10.1073/pnas.0636861100

Wells, J. A., and McClendon, C. L. (2007). Reaching for high-hanging fruit in drug discovery at protein-protein interfaces. Nature 450, 1001-1009. doi:10.1038/ nature 06526

Young, J. A. T., and Collier, R. J. (2007). Anthrax toxin: receptor binding, internalization, pore formation, and translocation. Annu. Rev. Biochem. 76, 243-265. doi:10.1146/annurev.biochem.75.103004.142728

Zelazo, D., Franchi, A., Allgower, F., Bulthoff, H. H., and Robuffo Giordano, P. (2012). Rigidity maintenance control for multi-robot systems. In N. Roy, N. Newman, and S. Srinivasa (473-480). Sidney, NSW: MIT Press. Available at: http://www.ist.uni-stuttgart.de/ zelazo/papers/2012g-ZelFraAlgBueRobpreprint.pdf

Conflict of Interest Statement: The authors declare that the research was conducted in the absence of any commercial or financial relationships that could be construed as a potential conflict of interest.

Copyright @ 2015 Di Paola, Platania, Oliva, Setola, Pascucci and Giuliani. This is an open-access article distributed under the terms of the Creative Commons Attribution License (CC BY). The use, distribution or reproduction in other forums is permitted, provided the original author(s) or licensor are credited and that the original publication in this journal is cited, in accordance with accepted academic practice. No use, distribution or reproduction is permitted which does not comply with these terms. 